\title{
The radial artery is protective in women and men following coronary artery bypass grafting - a substudy of the radial artery patency study
}

\author{
Derrick Y. Tam ${ }^{1,2}$, Saswata Deb ${ }^{1,2}$, Bao Nguyen ${ }^{1}$, Dennis T. Ko ${ }^{2,3}$, Reena Karkhanis ${ }^{1,2}$, Fuad Moussa ${ }^{1}$, \\ Jaclyn Fremes ${ }^{1}$, Eric A. Cohen ${ }^{3}$, Sam Radhakrishnan ${ }^{3}$, Stephen E. Fremes ${ }^{1,2}$; on behalf of the Radial \\ Artery Patency Study Investigators \\ ${ }^{1}$ Division of Cardiac Surgery, Schulich Heart Centre, Department of Surgery, Sunnybrook Health Sciences Centre, University of Toronto, Toronto, \\ Canada; ${ }^{2}$ Institute of Health Policy, Management and Evaluation, University of Toronto, Toronto, Canada; ${ }^{3}$ Division of Cardiology, Schulich Heart \\ Centre, Department of Medicine, Sunnybrook Health Sciences Centre, University of Toronto, Toronto, Canada \\ Correspondence to: Stephen E. Fremes, MD, MSc, FRCS. Dr. Bernard S. Goldman Chair in Cardiovascular Surgery, Schulich Heart Centre, \\ Sunnybrook Health Sciences Centre, 2075 Bayview Ave., Room H4 05, Toronto, ON, M4N 3M5, Canada. Email: stephen.fremes@sunnybrook.ca.
}

Background: Studies have demonstrated that female sex is an adverse risk factor in CABG. The primary aim of this study was to determine whether the radial artery (RA) was associated with reduced angiographic occlusion compared to the saphenous vein graft (SVG) stratified by sex in the multi-centered Radial Artery Patency Study (NCT00187356).

Methods: Between 1996-2001, 529 patients less than 80 years, with graftable triple-vessel disease underwent isolated CABG across 11 centers with late angiographic and clinical follow-up. The primary objective was to compare complete occlusion of RA and SVG with respect to sex. The secondary objective was to determine cumulative patency of both grafts along with predictors of late graft occlusion stratified by sex. The additional objective was to compare major adverse cardiac events (MACE, defined as cardiac mortality, myocardial infarction or re-intervention) between women and men.

Results: Of the 529 enrolled patients (13.4\% women), 269 (women: $\mathrm{n}=41,15.2 \%$ ) underwent late angiography at a mean of $7.7 \pm 1.5$ years after CABG. Women were older $(64.1 \pm 6.7$ versus $59.1 \pm 8.0$ years, $\mathrm{P}<0.01)$ with a higher rate of diabetes $(43.9 \%$ versus $28.5 \%, \mathrm{P}=0.05)$. Smoking history was less common (48.8\% versus $75.4 \%, \mathrm{P}<0.01$ ) while the mean number of grafts per patient were similar (women: $3.8 \pm 0.7$, men: $3.8 \pm 0.6, \mathrm{P}=0.65$ ). RA occlusions were lower than SVG in women (RA: 9.8\%, SVG: $26.8 \%, \mathrm{P}=0.05$ ) and in men (RA: $8.8 \%$, SVG: $17.1 \%, \mathrm{P}=0.01$ ). The rate of RA and SVG occlusion was not statistically different between women and men, and cumulative patency curves were also similar between sexes for the RA and study SVG. Multivariable modeling showed that having a RA (versus SVG) was protective in women [odds ratio (OR) 0.15, $\mathrm{P}=0.04$ ] and men: $(\mathrm{OR} 0.49, \mathrm{P}=0.02)$. MACE $(\mathrm{P}=0.15)$ and event-free cardiac survival (logrank $\mathrm{P}=0.14$ ) were similar between women and men.

Conclusions: Radial arteries are protective in both women and men with comparable burden of coronary disease and revascularization.

Keywords: Radial artery; coronary artery bypass grafting (CABG); sex differences; graft patency

Submitted Feb 01, 2018. Accepted for publication May 24, 2018.

doi: 10.21037/acs.2018.05.19

View this article at: http://dx.doi.org/10.21037/acs.2018.05.19 


\section{Introduction}

Coronary artery bypass grafting (CABG) remains an effective option for revascularization for those with extensive coronary artery disease. Patients benefit from the durability of this operation with improved survival and freedom from repeat revascularization compared to percutaneous coronary interventions. However, multiple observational studies have shown that despite adjustment for surgical risk using propensity matching or regression analysis, female sex remains a significant risk for both short and late mortality $(1,2)$. These differences in outcomes may be attributed to several factors such as differences in female coronary anatomy, revascularization strategy, and the choice of conduits (3). The importance of graft patency after CABG has been demonstrated in several studies and recently published randomized clinical trials. Graft occlusion or stenosis is associated with repeat revascularization events and mortality. As such, selecting conduits with excellent long-term patency remains an important cornerstone to successful coronary surgery. Understanding the impact of sex on graft patency may lend insight into mechanisms by which mortality outcomes can be improved for women undergoing CABG.

There is some evidence to suggest the use of bilateral internal thoracic arteries (BITA) may reverse the influence of sex on outcomes after CABG (4). However, BITA utilization remains low in North America and cited reasons for this include technical challenges in conduit harvesting, increased operative time, and concerns for deep sternal wound infections. Female sex has been identified as a risk factor for sternal infection in BITA patients (5). The length, diameter, and quality of the radial artery (RA) along with excellent wound healing at the site of harvest makes it an excellent conduit for bypass to non-left anterior descending (LAD) targets (6). Furthermore, the patency of the RA has been shown to be excellent and comparable to that of the left internal thoracic artery (LITA) $(7,8)$. In the Radial Artery Patency Study (RAPS), a longitudinal angiographic trial, in addition to the LITA to the LAD, patients were randomized to receive both a SVG and RA to either the left or right coronary arteries using a within patient randomization design whereby patients served as their own control $(9,10)$. Radial artery patency exceeded SVG patency at one year and beyond 5 years (approximately 7.5 years) postoperatively $(9,10)$. In multivariable analyses, female sex was associated with graft occlusion at the one year and the late angiographic assessment $(10,11)$. These findings suggest that female sex may impact graft patency in the long-term and that further research to characterize graft occlusion and stenosis may be important to improving conduit selection and late outcomes in women undergoing CABG.

Here, we explored differences in RA or SVG graft patency by sex from the multi-centred RAPS trial (NCT00187356). Our primary objective was to compare RA versus SVG graft occlusion in women and in men. Our secondary objective was to determine the cumulative patency of both grafts along with predictors of late graft occlusion stratified by sex. The tertiary objective was to compare all-cause mortality, major adverse cardiac events (MACE), defined as cardiac mortality, non-fatal myocardial infarction, and repeat revascularization, and the individual components of MACE between women and men.

\section{Methods}

This analysis is a substudy of the Radial Artery Patency Study (RAPS), which has been described extensively elsewhere $(9,10)$. In brief, 561 patients less than 80 years of age with triple vessel disease at 13 centers undergoing primary isolated non-emergent CABG were enrolled. In all patients, the LITA was grafted to the LAD. RAPS utilized a within patient randomization design: patients were randomized to have the RA grafted to either the right coronary artery (RCA) or the circumflex region; the SVG would be grafted to the opposing territory (circumflex or RCA). Each patient therefore received both study grafts and served as their own internal control. The target vessel for the RA or SVG was equal to or greater than $1.5 \mathrm{~mm}$ with a proximal stenosis of at least $70 \%$ by visual inspection on preoperative angiography. All grafts were harvested using an open technique and all CABGs were performed using an on-pump technique. All patients were approached to undergo invasive angiography after 5 years post CABG. Participating sites acquired angiograms digitally, and image sequences were transferred to the main study centre for centralized reading. Patients who declined invasive $\mathrm{X}$-ray angiography were offered the option to undergo computed topography angiography (CTA) and the protocol for this has been detailed elsewhere (9). In total, 440 patients underwent angiography 1 year post-operatively and 529 patients at nine Canadian centres participated in late follow-up (Figure 1). Of the 358 that remained alive and eligible for late angiography five years post-operatively, and 269 underwent late angiography (invasive $n=234$, CTA $n=35$ ). Each invasive angiogram was independently adjudicated in a blinded fashion by two interventional cardiologists; a third reviewer was 


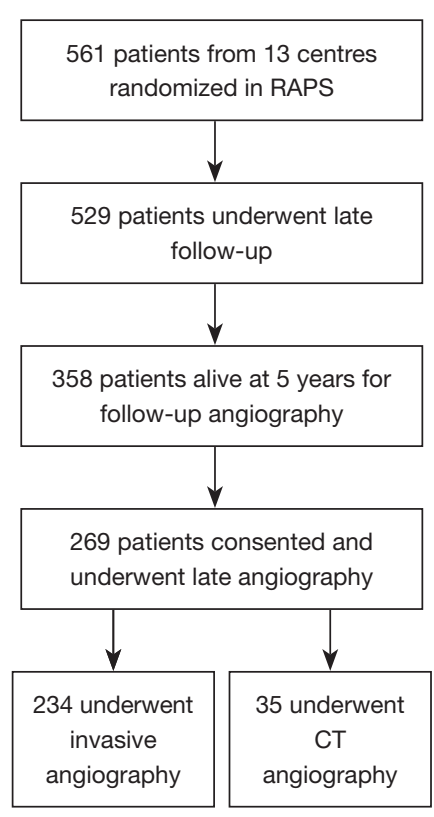

Figure 1 Patient flow diagram for RAPS Trial.

included in the case of disagreement. Study graft occlusion for this analysis was defined for complete graft occlusion.

\section{Statistics}

Baseline characteristics of patients who underwent longterm follow-up angiography were compared between women and men. Normality of continuous variables was assessed using the Kolmogorov-Smirnov test; a 2 -sample $t$-test and the Wilcoxon rank-sum test was used for parametric and nonparametric continuous variables, respectively. All categorical variables (reported as frequency and percentage) were compared using the Chi-square test or Fisher's exact $(\mathrm{n}<6)$. The McNemar's test was used to compare RA versus SVG occlusion within each sex. Comparisons of RA occlusion between women and men, and SVG occlusion between women and men were performed with the Chi-square test. Separate multivariable models were derived to identify predictors of graft occlusion in women and men. Generalized estimating equations (GEE) were used to determine the predictors of complete graft occlusion stratified by sex, as both study grafts were clustered within each patient-the dependent variable was graft occlusion (RA or SVG). Model predictors were selected a-priori based on clinical relevance; these included age at surgery in years, the use of a radial artery (versus study SVG), severity of target proximal native vessel stenosis $(70-89 \%$ versus $>90 \%)$, diabetes, hypertension, history of smoking, hyperlipidemia, creatinine (umol/L), and peripheral vascular disease. These covariates were tested for multicollinearity using tolerance statistics; a tolerance value of $<0.4$ was used as the cutpoint for the presence of multicollinearity. All-cause mortality, MACE, and MACE events were compared between women and men using the Chi-square test and using log-rank statistics. All analyses were performed using SAS (version 9.4; SAS Institute, Inc., Cary, NC, USA). A two tailed $\mathrm{P}$ value less than 0.05 was considered statistically significant.

\section{Results}

\section{Pre-operative patient and angiographic characteristics}

Of the 561 patients randomized in RAPS, there were 269 patients [female, $41(16.2 \%)$, male, $228(84.8 \%)$ ] who underwent late angiography at a mean follow-up of $7.7 \pm 1.5$ years. Women were older $(64.1 \pm 6.7$ versus $59.1 \pm 8.0$; $\mathrm{P}<0.01)$, more likely to be diabetic, and more likely hypertensive compared to male patients (Table 1). However, more men were smokers and the baseline creatinine was higher in the male population. Preoperative angiographic characteristics are summarized in Table 1 . There were no differences in the target vessel stenosis by conduit type (RA target vessel versus SVG target vessel) or by coronary system (right versus circumflex target vessel) as assessed by visual inspection at the time of invasive angiography between women and men. The mean number of grafts per patient were similar between women and men (W: $3.8 \pm 0.7, \mathrm{M}$ : $3.8 \pm 0.6, \mathrm{P}=0.65)$.

\section{Graft occlusion}

In both women $(26.8 \%$ versus $9.8 \% ; \mathrm{P}=0.05)$ and men $(17.1 \%$ versus $8.8 \% ; \mathrm{P}=0.01)$, the rate of $\mathrm{SVG}$ occlusion was significantly higher than that of RA occlusion (Figure $2 A$ ). Between women and men, there was no difference in rates of RA occlusion (9.8\% versus $8.8 \% ; \mathrm{P}=0.77$ ), SVG occlusion $(26.8 \%$ versus $17.1 \% ; \mathrm{P}=0.14)$ or any occlusion (34.2\% versus $25.4 \% ; \mathrm{P}=0.25$ ) (Figure $2 B$ ). There was no difference in the cumulative patency of RA graft between women and men (Figure $3 A, \log$ rank $\mathrm{P}=0.69$ ) — the curves were overlapping. When cumulative patency of SVG was examined over time, there was no significant difference in graft patency between women and men, although results in men were consistently numerically higher (Figure $3 B, \log$ 
Table 1 Clinical characteristics of patients who underwent long-term follow-up angiography $(7.7 \pm 1.5$ years $)$

\begin{tabular}{|c|c|c|c|}
\hline Characteristic & Women $(\mathrm{N}=41)$ & Men $(\mathrm{N}=228)$ & $P$ value* \\
\hline Age $>70$ years, $n(\%)$ & $8(19.5)$ & $24(10.5)$ & 0.10 \\
\hline Elective surgery, n (\%) & $23(56.1)$ & $153(67.1)$ & 0.17 \\
\hline Previous myocardial infarction, $\mathrm{n}(\%)$ & $15(36.6)$ & $109(47.8)$ & 0.18 \\
\hline CCS class of angina, $\mathrm{n}(\%)$ & & & 0.20 \\
\hline 1 & $1(2.4)$ & $3(1.3)$ & \\
\hline 2 & $5(12.2)$ & $54(23.7)$ & \\
\hline 3 & $20(48.8)$ & $117(51.3)$ & \\
\hline Hypertension, n (\%) & $27(65.9)$ & $94(41.2)$ & $<0.01$ \\
\hline Hypercholesterolemia, n (\%) & $32(78.0)$ & $157(68.9)$ & 0.15 \\
\hline Smoking history, n (\%) & $20(48.8)$ & $172(75.4)$ & $<0.01$ \\
\hline Creatinine, $\mu \mathrm{mol} / \mathrm{L}$ (mean $\pm \mathrm{SD}$ ) & $82.1 \pm 15.5$ & $94.4 \pm 17.2$ & $<0.01$ \\
\hline Peripheral vascular disease, $\mathrm{n}(\%)$ & $0(0)$ & $16(7.0)$ & 0.14 \\
\hline Left ventricular grade, $\mathrm{n}(\%)^{\dagger}$ & & & 0.37 \\
\hline 1 & $25(61.0)$ & $108(47.4)$ & \\
\hline 2 & $16(39.0)$ & $115(50.4)$ & \\
\hline
\end{tabular}

Target-vessel stenosis, $\mathrm{n}(\%)$

Radial-artery target vessel

70-89\% stenosis

$21(51.2)$

$\geq 90 \%$ stenosis

$20(48.8)$

Saphenous-vein target vessel

$70-89 \%$ stenosis

$16(39.0)$

$\geq 90 \%$ stenosis

$25(61.0)$

$\begin{array}{ll}84(36.8) & 0.08 \\ 144(63.2) & 0.08 \\ & \\ 78(34.2) & 0.56 \\ 150(65.8) & 0.56\end{array}$

*, P value represents an analysis of statistical significance between the male and female cohorts. ${ }^{\dagger}$, according to this scale, grade 1 indicates an estimated global left ventricular ejection fraction (LVEF) of $\geq 50 \%$, grade 2 indicates LVEF of 35-49\%, grade 3 indicates LVEF of $20-34 \%$, and grade 4 indicates LVEF of $\leq 20 \%$. CCS, Canadian Cardiovascular Society.

rank $\mathrm{P}=0.19)$.

\section{Predictors of graft occlusion by sex}

We compared nine predictors for graft occlusion in a multi-variable GEE model (Table 2). History of smoking was a significant predictor of increased graft failure in men (OR 1.98; 95\% CI: 1.27, 3.09; P<0.01). Similarly, elevated creatinine was also a significant predictor of increased graft occlusion in men (OR 1.18; 95\% CI: 1.02, 1.36; $\mathrm{P}=0.02$ ). 

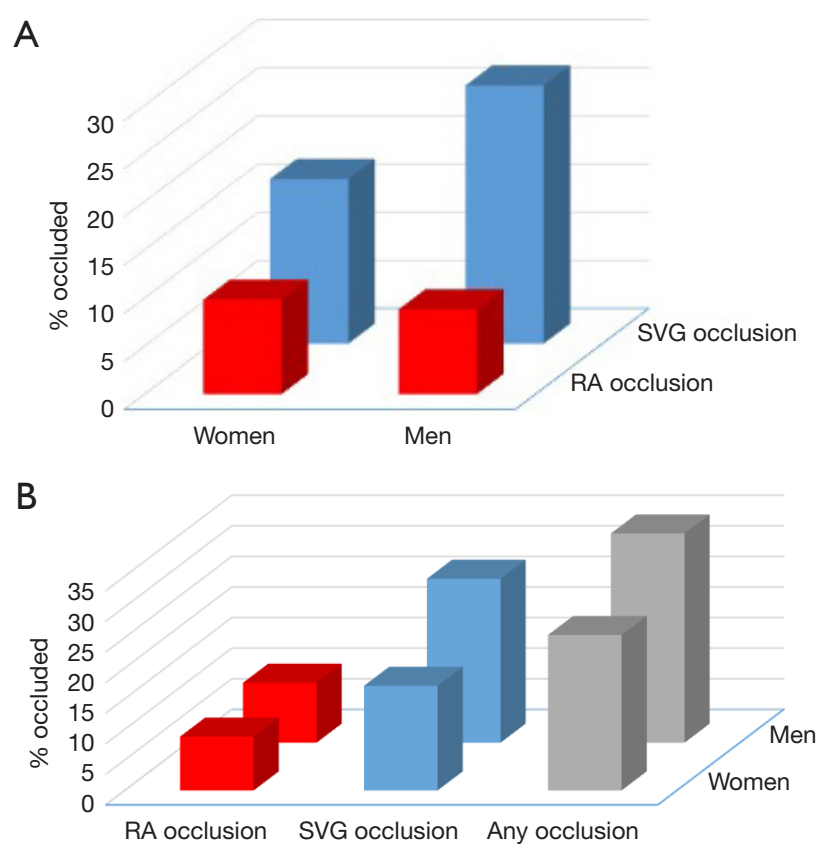

Figure 2 Graft occlusion and sex. (A) Comparison of graft occlusion of the radial artery (RA) versus saphenous vein graft (SVG) stratified by sex. The rate of SVG occlusion was higher than the rate of RA occlusion in women $(26.8 \%$ versus $9.8 \%, \mathrm{P}=0.05)$ and in men $(17.1 \%$ versus $8.8 \%, \mathrm{P}=0.01)$. (B) Comparison of graft occlusion between women and men. There was no difference in the occlusion rate of the radial artery (RA, 9.8\% versus $8.8 \%$, $\mathrm{P}=0.77$ ), saphenous vein graft (SVG, $26.8 \%$ versus $17.1 \%, \mathrm{P}=0.14$ ), or occlusion of any graft (34.2\% versus $25.4 \%, \mathrm{P}=0.25)$ between women and men.

The degree of target vessel stenosis was protective of graft occlusion in women (OR: 0.13; 95\% CI: 0.02, 0.67; $\mathrm{P}=0.02$ ). In both women (OR: 0.15; 95\% CI: 0.03, 0.91; $\mathrm{P}=0.04)$ and men (OR: $0.49,95 \%$ CI: 0.26, 0.90; $\mathrm{P}=0.02$ ), a RA graft was protective from graft occlusion when controlling for other factors.

\section{Clinical endpoints by sex}

There was a significant difference in all-cause mortality that was higher in men $(12.9 \%)$ than women $(2.8 \%$, $\mathrm{P}<0.01)$. Similarly, cardiac death was higher in men $(5.7 \%)$ compared to women $(0 \%, \mathrm{P}=0.04)$. There was no difference in the occurrence of MI (2.0\% versus $1.4 \%)$, need for repeat revascularization by PCI $(5.0 \%$ versus $5.6 \%)$ and major adverse cardiac events (11.8\% versus $5.6 \%$ ) between men and women (Figure 4). Cumulative cardiac event free
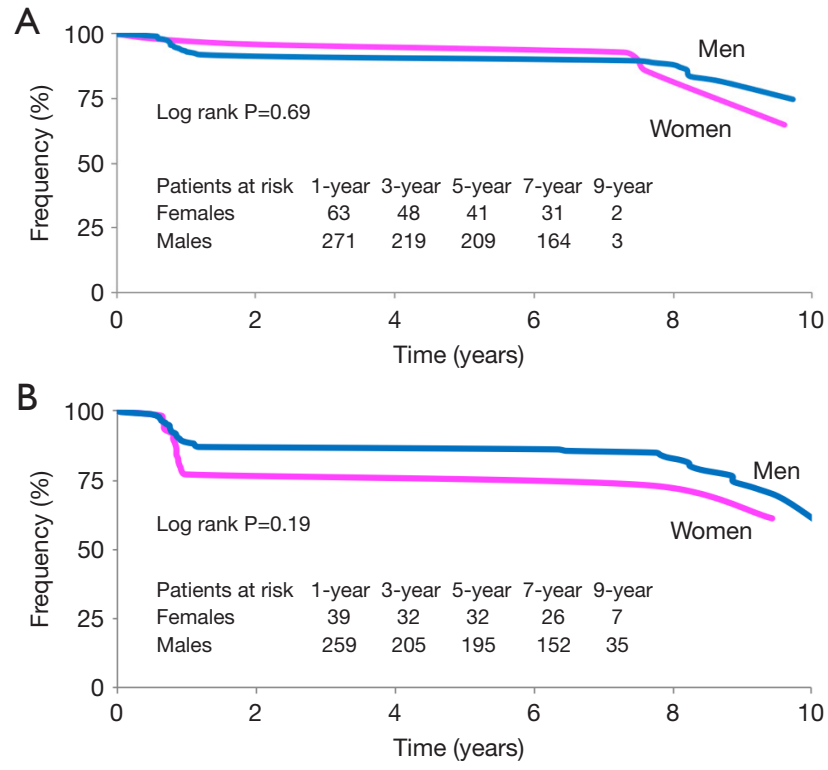

Figure 3 Long-term patency of radial arteries and saphenous vein grafts in women and men. (A) Cumulative patency of radial artery grafts of women (pink) and men (blue). Patency was defined as the absence of a complete graft occlusion. The curves were overlapping. (B) Cumulative patency of saphenous vein grafts of women (pink) and men (blue). Patency was defined as the absence of a complete graft occlusion. The patency of saphenous vein grafts was numerically higher for men.

survival was similar between men and women (Figure 5; log rank $\mathrm{P}=0.14)$.

\section{Discussion}

The most important finding of this substudy of the RAPS trial was that the use of the radial artery as a bypass conduit for grafts to the right coronary or circumflex territories was protective in both women and men. Higher-grade proximal native vessel stenosis was found to be protective against late graft occlusion in women.

These findings are pertinent in the management of advanced $\mathrm{CAD}$ in women with implications on addressing the enhanced surgical risk. Early clinical trials of surgical revascularization enrolled mostly men. Later studies suggested that CAD in women may be under-diagnosed or misdiagnosed, and that women were less frequently evaluated with invasive angiography or referred for CABG surgery compared to men of similar profile. Contemporary meta-analysis of observational studies in CABG has 
Table 2 Predictors for occlusion in females and males

\begin{tabular}{|c|c|c|c|c|}
\hline Variable & \multicolumn{2}{|c|}{ Women } & \multicolumn{2}{|c|}{ Men } \\
\hline Age & $0.95(0.84-1.07)$ & 0.40 & $1.00(0.96-1.03)$ & 0.87 \\
\hline Diabetes mellitus & $0.85(0.25-2.86)$ & 0.80 & $1.16(0.65-2.06)$ & 0.61 \\
\hline Radial artery & $0.15(0.03-0.91)$ & 0.04 & $0.49(0.26-0.90)$ & 0.02 \\
\hline Smoking history & $0.40(0.15-1.03)$ & 0.06 & $1.98(1.27-3.09)$ & $<0.01$ \\
\hline Hypercholesterolemia & $0.29(0.05-1.69)$ & 0.17 & $0.95(0.52-1.71)$ & 0.85 \\
\hline Creatinine & $1.22(0.84-1.76)$ & 0.29 & $1.18(1.02-1.36)$ & 0.02 \\
\hline Peripheral vascular disease & 0 & 0 & $0.73(0.26-2.09)$ & 0.56 \\
\hline
\end{tabular}

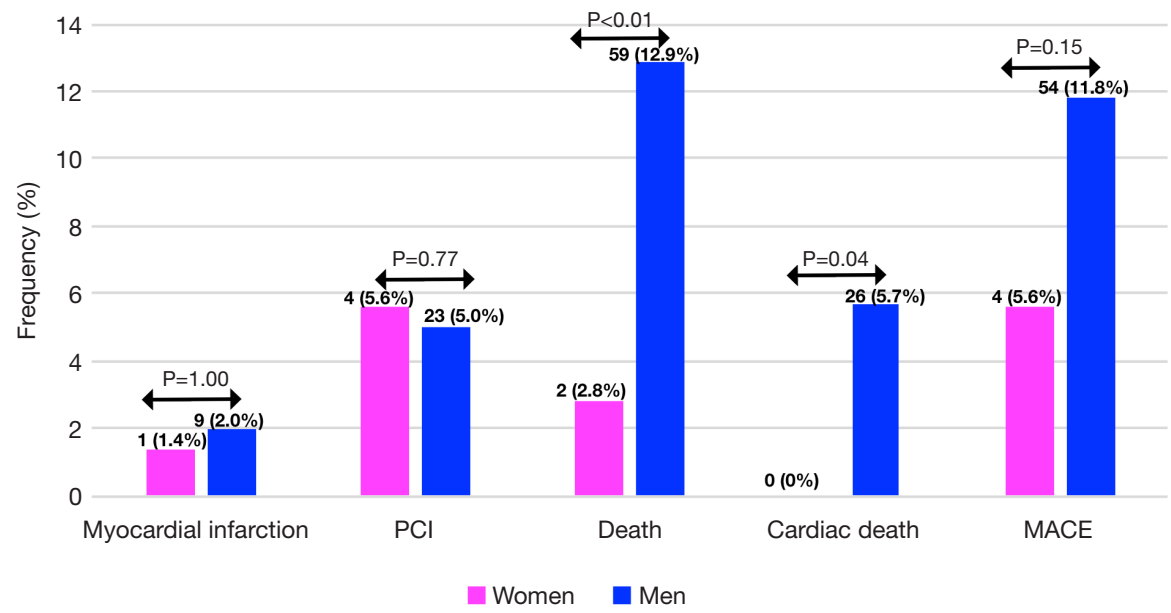

Figure 4 Late myocardial infarction, percutaneous coronary intervention (PCI), all-cause mortality, cardiac death and major adverse cardiac events (MACE, cardiac death, late myocardial infarction and repeat revascularization) in both women (pink) and men (blue)

shown both early and late mortality to be worse in women compared to men (2). The literature has consistently shown women are more likely to present at an advanced stage of disease with greater severity of symptoms than their male counterparts and with a greater comorbidity burden at the time of surgery (12). The pattern of distribution of coronary artery disease can exhibit marked sex differences with certain higher grade disease patterns seen in women (13). Moreover, earlier studies have demonstrated a lower use of the ITA as a primary graft in women and lower use of a second arterial conduit compared to men, that may account for some of the additional risk seen in women $(14,15)$. Kurlansky et al. compared late survival in a retrospective analysis of 602 and 328 propensity matched pairs of men and women that underwent BITA or single ITA grafting respectively (4). The study showed that late survival amongst men and women matched to BITA was comparable at 20 years $(31.3 \%$ versus $30.1 \% \mathrm{P}=0.790)$ despite a trend towards lower perioperative survival in women $(5.8 \%$ versus $3.5 \% ; \mathrm{P}=0.055)$ when treated with single ITA compared to men.

An interesting finding in our analysis was the observation that overall survival was better in women than men, contrary to results reported in the literature. In the RAPS trial, all patients enrolled had graftable targets with a high number of grafts performed in both women 


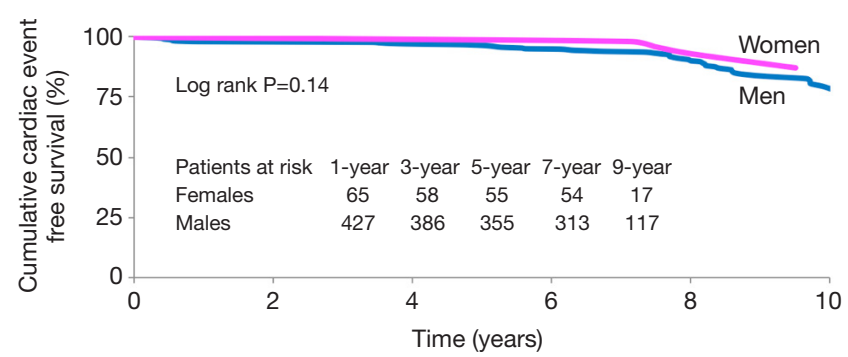

Figure 5 Cumulative major adverse cardiac event (MACE) free survival for women (pink) and men (blue). MACE was defined as cardiac mortality, non-fatal myocardial infarction, and repeat revascularization.

and men. Women usually have less grafts performed per patient, after controlling for the number of diseased vessels (16), and suggests that women are more likely to have incomplete revascularization. Thus, the improved outcomes seen in our study may in part be due to more complete revascularization than is usually achieved in females, and the use of two arterial grafts in all study patients. In a propensity matched analysis of females receiving a RA compared to those receiving SVG at the time of CABG, late Kaplan-Meier estimated survival was higher in women who received RA at 15-year followup $(70 \%$ versus $58 \%)(17)$. We acknowledge that the differences were unadjusted and the numbers of individual MACE events are small-when the composite MACE outcome was compared between women and men, the Kaplan Meier curves were very similar (Figure 5).

Consistent with the literature, we showed that the patency was higher with RA compared to SVG; however, in this study we showed that was true in women as well as men. Late follow-up of angiographic outcome studies for the RA have shown consistently high patency rates of greater than $80 \%$ at later than seven years of follow-up $(8,18)$. In a follow-up of their first 100 patients that underwent CABG with RA grafting, Gaudino et al. showed in 36 patients at 20 years of follow-up a patency rate of $84.4 \%$, comparable to the rate of LITA patency of $93.9 \%$ (7). In contrast, SVG patency was reduced at $45.1 \%$ in that same study. In a metaanalysis of RCTs comparing RA or SVG, graft occlusion favored RA at four-year follow-up (19).

The impetus for this substudy was earlier findings from previous secondary analyses that suggested higher graft occlusion results with women in RAPS. An exploratory analysis of the 440 patients with one-year angiography, revealed that female sex was a multivariable risk factor for graft occlusion (relative risk 1.78, 95\% CI: 1.11-2.84, $\mathrm{P}=0.02$ ), although there was a graft type by sex interaction (RA occlusion similar between women and men, while SVG occlusion was increased in women) (10). A similar exploratory analysis after late angiography in 269 patients revealed that female sex was a multivariable risk factor for graft occlusion (OR 2.23, 95\% CI: 1.14-4.38, $\mathrm{P}=0.02$ ) (11). Graft occlusion (of the RA or SVG) was higher in women ( $34.2 \%$ versus $25.4 \%$, Figure $2 B$ ), mainly because of increased SVG occlusion.

\section{Limitations}

This study must be interpreted in the context of some important limitations. Any post-hoc subgroup analysis must be considered hypothesis generating or exploratory. In our study, the number of men included was more than fivefold higher than women; this limited sample size may be underpowered to detect differences in clinical outcomes. Furthermore, baseline characteristics were not identical between the groups and we did not adjust for potential known confounders between the groups. Differences in baseline characteristics may explain the profound difference in all-cause mortality between men and women. However, our intergraft comparisons between RA and SVG stratified by sex is not likely impacted by bias, given the within patient randomization design utilized. We also recognize that we have not considered other arterial grafts such as the right ITA. It remains unclear whether a bilateral ITA strategy is superior to that of a LITA/RA strategy $(20,21)$.

\section{Conclusions}

The use of the radial artery as a second arterial conduit in addition to the use of the internal thoracic artery is appropriate and protective in both women and men with advanced coronary artery disease.

\section{Acknowledgements}

Funding: Dr. Tam is supported by the Ontario Ministry of Health Clinician Investigator's Program (Toronto, Ontario). Dr. Ko is supported by a Mid-Career Award by the Heart and Stroke Foundation of Canada (Toronto, Ontario). Dr. Fremes is supported by the Bernard S. Goldman Chair in Cardiovascular Surgery (Toronto, Ontario). This study was funded in part by the Canadian Institutes of Health Research (CIHR) grant MCT 52681 
and the Bernard Goldman Chair in Cardiovascular Surgery.

\section{Footnote}

Conflicts of Interest: The authors have no conflicts of interest to declare.

\section{References}

1. Alam M, Lee VV, Elayda MA, et al. Association of gender with morbidity and mortality after isolated coronary artery bypass grafting. A propensity score matched analysis. Int J Cardiol 2013;167:180-4.

2. Alam M, Bandeali SJ, Kayani WT, et al. Comparison by meta-analysis of mortality after isolated coronary artery bypass grafting in women versus men. Am J Cardiol 2013;112:309-17.

3. Bolooki H. The controversy in clinical results among men and women after coronary bypass operation. J Am Coll Cardiol 2007;49:1559-60.

4. Kurlansky PA, Traad EA, Dorman MJ, et al. Bilateral internal mammary artery grafting reverses the negative influence of gender on outcomes of coronary artery bypass grafting surgery. Eur J Cardiothorac Surg 2013;44:54-63.

5. Gatti G, Dell'Angela L, Barbati G, et al. A predictive scoring system for deep sternal wound infection after bilateral internal thoracic artery grafting. Eur J Cardiothorac Surg 2016;49:910-7.

6. Raza S, Sabik JF, Masabni K, et al. Surgical revascularization techniques that minimize surgical risk and maximize late survival after coronary artery bypass grafting in patients with diabetes mellitus. J Thorac Cardiovasc Surg 2014;148:1257-64; discussion 1264-6.

7. Gaudino M, Tondi P, Benedetto U, et al. Radial Artery as a Coronary Artery Bypass Conduit: 20-Year Results. J Am Coll Cardiol 2016;68:603-10.

8. Tranbaugh RF, Dimitrova KR, Friedmann P, et al. Coronary artery bypass grafting using the radial artery: clinical outcomes, patency, and need for reintervention. Circulation 2012;126:S170-5.

9. Deb S, Cohen EA, Singh SK, et al. Radial artery and saphenous vein patency more than 5 years after coronary artery bypass surgery: results from RAPS (Radial Artery Patency Study). J Am Coll Cardiol 2012;60:28-35.

10. Desai ND, Cohen EA, Naylor CD, et al. A randomized comparison of radial-artery and saphenous-vein coronary bypass grafts. N Engl J Med 2004;351:2302-9.

11. Deb S, Singh SK, Moussa F, et al. The long-term impact of diabetes on graft patency after coronary artery bypass grafting surgery: a substudy of the multicenter Radial Artery Patency Study. J Thorac Cardiovasc Surg 2014;148:1246-53; discussion 1253.

12. Blasberg JD, Schwartz GS, Balaram SK. The role of gender in coronary surgery. Eur J Cardiothorac Surg 2011;40:715-21.

13. Chiha J, Mitchell P, Gopinath B, et al. Gender differences in the severity and extent of coronary artery disease. Int J Cardiol Heart Vasc 2015;8:161-6.

14. Fukui T, Tabata M, Manabe S, et al. Angiographic outcomes of right internal thoracic artery grafts in situ or as free grafts in coronary artery bypass grafting. J Thorac Cardiovasc Surg 2010;139:868-73.

15. Ennker IC, Albert A, Pietrowski D, et al. Impact of gender on outcome after coronary artery bypass surgery. Asian Cardiovasc Thorac Ann 2009;17:253-8.

16. Hassan A, Chiasson M, Buth K, et al. Women have worse long-term outcomes after coronary artery bypass grafting than men. Can J Cardiol 2005;21:757-62.

17. Dimitrova KR, Hoffman DM, Geller CM, et al. Radial artery grafting in women improves 15 -year survival. J Thorac Cardiovasc Surg 2013;146:1467-73.

18. Achouh P, Isselmou KO, Boutekadjirt R, et al. Reappraisal of a 20-year experience with the radial artery as a conduit for coronary bypass grafting. Eur J Cardiothorac Surg 2012;41:87-92.

19. Cao C, Manganas C, Horton M, et al. Angiographic outcomes of radial artery versus saphenous vein in coronary artery bypass graft surgery: a meta-analysis of randomized controlled trials. J Thorac Cardiovasc Surg 2013;146:255-61.

20. Nasso G, Coppola R, Bonifazi R, et al. Arterial revascularization in primary coronary artery bypass grafting: Direct comparison of 4 strategies--results of the Stand-in-Y Mammary Study. J Thorac Cardiovasc Surg 2009;137:1093-100.

21. Gaudino M, Cellini C, Pragliola C, et al. Arterial versus venous bypass grafts in patients with in-stent restenosis. Circulation 2005;112:I265-9.

Cite this article as: Tam DY, Deb S, Nguyen B, Ko DT, Karkhanis R, Moussa F, Fremes J, Cohen EA, Radhakrishnan S, Fremes SE; on behalf of the Radial Artery Patency Study Investigators. The radial artery is protective in women and men following coronary artery bypass grafting-a substudy of the radial artery patency study. Ann Cardiothorac Surg 2018;7(4):492-499. doi: 10.21037/acs.2018.05.19 